\title{
EXTREMA OF PRINCIPAL CURVATURE AND SYMMETRY
}

\author{
by J. W. BRUCE and F. TARI*
}

(Received 19th December 1994)

\begin{abstract}
In this paper we show that away from umbilic points certain measures of the local reflectional symmetry of a surface in Euclidean 3-space are detected by the extrema of the sectional curvatures along lines of curvature. There are two types of reflectional symmetry, with one detected by the contact between the surface and spheres, and in this case the result is due to Porteous and is 20 years old. We show that an analogous result remains true for the second type of symmetry.
\end{abstract}

1991 AMS Classification Numbers: 58C 53A

\section{Introduction}

Consider a smooth surface in Euclidean 3-space. At each non-umbilic point $\boldsymbol{p}$ there are two (orthogonal) principal directions with corresponding principal curvatures, which are smooth functions in a neighbourhood of $p$. Away from umbilic points the principal directions integrate up to yield lines of curvature, and we can consider the restriction of the principal curvatures to these lines of curvature (two functions for each such line). What we seek is an interpretation of extrema of these functions. Generally these will occur at isolated points, and as we vary the lines these points will sweep out curves on the surface of some geometric significance. When the curvature function is restricted to its corresponding line of curvature these are the important ridge curves of Porteous $([8$, 9]) which arise in his study of surfaces using the family of distance-squared functions. In this paper we shall show that the union of the extrema of the curvature function when restricted to the other principal curve is the so-called sub-parabolic line first discussed in [14] and [2], and also in [7]. Indeed the approach taken in the first two of these works, that of studying the geometry of the surface using a family of fold mappings (first introduced in [1]), produces both the ridge and sub-parabolic points on a surface, in a more geometrically significant way than as the union of the given extrema. However their interpretation in terms of classical differential geometric invariants is of interest, and of help when one wishes to determine these loci on a given surface. (Ridge and subparabolic points on a surface are of considerable interest to those in the field of computer vision, see, $[3,4,11,12]$.)

More significantly we prove another analogue of a result of Porteous, namely that one can detect more degenerate singularities by considering the contact of the sub-

* The second author was supported by the Esprit grant VIVA while this paper was in preparation. 
parabolic curves with the line of curvature. The basic insight to be deduced from this work is that these extrema locate (some of) the points on the surface of local infinitesimal reflectional symmetry.

As a general background for the singularity theory approach to differential geometry we suggest [13] and [10], and as a text for the (small amount of classical differential geometry we shall need [5]. We shall use subscript notation for partial derivatives.

The authors are grateful to Ian Porteous and Professor Thirion for suggesting that the sub-parabolic curves on a surface can be interpreted in this way; we understand that the latter has an alternative proof.

\section{Ridge and sub-parabolic sets}

In what follows we shall suppose that we have a smooth surface $X$ in Euclidean 3-space, and a point $p$ fixed on that surface; we will consider $X$ near $p$ as being parametrised by some germ $i: \mathbf{R}^{2}, 0 \rightarrow \mathbf{R}^{3}, 0$. We shall suppose that $p$ is a non-umbilic, consequently there is an orthogonal net of lines of curvature near $p$, and a pair of welldefined curvature functions. We next fix a principal direction at $p$. We can fold the surface across the plane containing the normal and the given principal direction, as described in [2], [1]. In other words if we choose co-ordinates in such a way that the point $p$ is at the origin, the normal is in the $z$-direction and the given principal direction is the $y$-axis, then we restrict the fold mapping $f(x, y, z)=\left(x^{2}, y, z\right)$ to the surface. This yields a map-germ $f \circ i: \mathbf{R}^{2}, 0 \rightarrow \mathbf{R}^{3}, 0$ and it is the $\mathscr{A}$-type of this germ that we will be considering. There are two basic families of such germs which shall interest us. A germ is said to be of type $B_{k}, k \geqq 1$ (resp. $S_{k}, k \geqq 2$ ) if it is $\mathscr{A}$-equivalent to $\left(x, y^{2}, x^{2} y \pm y^{2 k+1}\right.$ ) (resp. $\left(x, y^{2}, y^{3} \pm x^{k+1} y\right)$ ). The subscript $k$ is a measure of the degree of complication of the singularity. (Here we are using the notation of Mond [6].) The subparabolic points on the surface are those where the folding map has $S_{\geq 2}$ singularities, or equivalently the corresponding point on the focal set is parabolic. The results of Porteous on the other hand are concerned with the contact of the surface with tangent spheres. Here we select the centre of curvature corresponding to the given principal direction (say $(0,0, \alpha))$ and consider the distance-squared function from $(0,0, \alpha)$, given by $d(x, y, z)=x^{2}+y^{2}+(z-\alpha)^{2}$. The contact is then measured by the composite $d \circ i: \mathbf{R}^{2}, 0 \rightarrow \mathbf{R}, 0$; the relevant equivalence relation here is contact or $\mathscr{K}$-equivalence. Thus the given principal direction has a curvature function, a line of curvature, a folding map and a distance-squared function associated with it. The simplest family of singularities here are the $A_{k}, k \geqq 1$, which are those $\mathscr{K}$-equivalent to $x^{2} \pm y^{k+1}$. We can now state the first of our two theorems.

Theorem 2.1. (i) (Porteous [9]). The contact between the surface and the corresponding sphere of curvature is of type $A_{\geqq 3}$ (i.e. we have a ridge) if and only if the restriction of the corresponding curvature function to the line of curvature has an extremum at p. (In terms of the folding mappings $A_{3}$ contact occurs if and only if we have had a $B_{\geqq 2}$ singularity for the corresponding folding.)

(ii) The folding map has an $S_{\geq 2}$ if and only if the restriction of the corresponding curvature function to the other line of curvature through $p$ has an extremum at $p$. 
Proof. The proof of the required result is, as one might expect, a straightforward calculation. So let $E, F, G$ and $e, f, g$ be the coefficients of the first and second fundamental form of a surface $X$ in 3-space. The principal curvatures at any point are then given as the zeros of the quadratic equation

$$
t^{2}\left(E G-F^{2}\right)+t(2 F f-E g-G e)+\left(e g-f^{2}\right)=0 .
$$

If the parameters on the surface are $u$ and $v$ the left hand side is a function, say $P$, of the variables $u, v, t$. Let $t_{0}$ be a principal curvature at the non umbilic point $\left(u_{0}, v_{0}\right)$, and suppose for the moment that at the point in question the derivative $P_{t}$ is non-zero if and only if the point in question is not an umbilic. Supposing this is so we can apply the implicit function theorem and write $t$ locally as a function $T$ of $u$ and $v$, with

$$
P(u, v, T(u, v))=0 .
$$

Suppose now that the $u$ and $v$ directions are principal. Then we seek to interpret the vanishing of the derivatives $T_{u}$ and $T_{v}$. This is clearly equivalent to the vanishing of $P_{u}$, respectively $P_{v}$.

We now seek to relate these conditions to those concerning the folding map. One way to do this is to write the surface in Monge form, that is as a graph $(u, v, h(u, v))$ where $h$ has no linear or constant part. One then seeks to interpret both conditions in terms of the coefficients in the Taylor expansion of $h$. We start with those concerning the extrema.

We shall see that both sets of conditions only involve the quadratic and cubic terms of $h$. Since we are at a non-umbilic we write $h$ in the form

$$
a_{0} u^{2}+a_{2} v^{2}+C(u, v)+\mathrm{O}(4)
$$

where $C$ is cubic, and $O(4)$ denotes terms vanishing to order 3 . Consequently the $u$ and $v$ directions are principal, $a_{0} \neq a_{2}$, and the principal curvatures are $2 a_{0}$ and $2 a_{2}$. It is now a straightforward calculation to show that to order 1 we can write $E=1, F=0$, $G=1, e=2 a_{0}+C_{u u}, f=C_{u v}, g=2 a_{2}+C_{v v}$. It follows that at $u=v=0$ we have

$$
P_{u}=t\left(-C_{u v v}-C_{u u u}\right)+2\left(a_{0} C_{u v v}+a_{2} C_{u u u}\right)
$$

Setting $t=2 a_{0}$ we find that $P_{u}=2\left(a_{2}-a_{0}\right) C_{\text {uus }}$ while if $t=2 a_{2}$ this partial derivative is $2\left(a_{0}-a_{2}\right) C_{u v v}$. Thus the partial derivative $P_{u}$ vanishes at $\left(0,0,2 a_{0}\right)$ (resp. $\left.\left(0,0,2 a_{2}\right)\right)$ if and only if $C_{u u u}$ (respectively $C_{u v v}$ ) is zero. (Note that the partial derivative $P_{t}$ evaluated at $\left(0,0,2 a_{2}\right)$ (resp. $\left.\left(0,0,2 a_{0}\right)\right)$ is $2\left(a_{2}-a_{0}\right)$ (resp. $\left.2\left(a_{0}-a_{2}\right)\right)$ and so does not vanish away from umbilics.)

We now turn to the folding map. The import of [2] is that at a non-umbilic a surface has maximum reflectional symmetry across the pair of planes containing the normal and the principal directions. Writing the surface in the above form $(u, v, h(u, v))$ this symmetry is measured by the $\mathscr{A}$-type of the singularity $(u, v) \mapsto\left(u^{2}, v, h(u, v)\right)$ (for the plane 
containing the $v$-direction) and $(u, v) \mapsto\left(u, v^{2}, h(u, v)\right)$ (for the plane containing the $u$-direction). We can detect the relevant types of singularity from the 3-jets of these maps. In the first case this 3-jet is

$$
\left(u^{2}, v, a_{0} u^{2}+a_{2} v^{2}+\left(b_{0} u^{3}+b_{1} u^{2} v+b_{2} u v^{2}+b_{3} v^{3}\right)\right)
$$

Straightforward changes of co-ordinates in the target reduce this to

$$
\left(u^{2}, v, b_{0} u^{3}+b_{2} u v^{2}\right)
$$

If the product $b_{0} b_{2}$ does not vanish one can reduce to the normal form $\left(u^{2}, v, u^{3} \pm u v^{2}\right)$ which is 3-determined and is of type $B_{1}$. This singularity represents the generic reflectional local symmetry of a surface. Thus more degenerate symmetry corresponds to the vanishing of $b_{0}$ and/or $b_{2}$. Assuming that both terms do not vanish simultaneously we find that we have a germ in the $S$ stem if $b_{2}=0$, and of type $B_{\geqq 2}$ if $b_{0}=0$. Of course these are exactly the conditions that $C_{u v v}=0$ and $C_{u u u}=0$. It is to be noted that we have a $B_{\geqq 2}$ of the folding map if and only if we are at a ridge point of the surface. This is a straightforward calculation. This establishes the result.

\section{Tangency}

We now turn our attention to the question of interpreting tangency of the ridge (respectively sub-parabolic curve) with the lines of curvature. Again the result in the first case is due to Porteous. Note from [2] the folding map will have an $S_{\geqq 3}$ on the surface when the focal set has a cusp of Gauss.

Theorem 3.1. (i) (Porteous [9]) If $p$ is a point of the ridge set, and the ridge curve is tangent to the corresponding line of curvature (equivalently the curvature along the corresponding line of curvature has a degenerate extremum), then the singularity of the contact with the corresponding sphere of curvature is of type $A_{\geqq 4}$.

(ii) If $p$ is a point of the sub-parabolic curve where it is tangent to the corresponding line of curvature, then the corresponding folding map is of type $S_{\geqq 3}$.

Remark 3.2. (i) Although the ridge points are detected by the family of folding mappings the $A_{4}$ points on that curve are not.

(ii) Note that in the second case the tangency is not equivalent to the corresponding extremum of the curvature function being degenerate.

Proof. We shall outline the proof in both cases: they are the result of fairly messy calculations. We shall use the same notation as above for our surface, except that now $C$ will denote the cubic and quartic parts of the height function $h$. In case (i) (the case $t=2 a_{0}$ above) we need to consider the second derivative of the function $T$ along a principal direction. To obtain this we need to find some further information on the 2-jet 
of the line of curvature in the $(u, v)$-plane. Using the standard equation for the principal directions

$$
(E f-e F) d u^{2}+(E g-e G) d u d v+(F g-f G) d v^{2}=0
$$

it is not hard to show that the 2-jet is of the form $\left(s+A s^{2}, B s^{2}\right)$ where $B=C_{u u v} / 4\left(a_{0}-a_{2}\right)$. Differentiating the function $T$ twice along this curve and evaluating at $s=0$ we obtain $T_{u u}+2 A T_{u}+2 B T_{v}$ (all at $\left.(u, v, t)=\left(0,0,2 a_{0}\right)\right)$ and in the case of interest we have $T_{u}=0$. On the other hand the second derivative $T_{u u}$ can be deduced from the equation $P_{u u}+$ $2 P_{u t} T_{u}+P_{t} T_{u u}=0$ obtained by differentiating the equation $P(u, v, T(u, v))=0$ twice. Putting these results together we find that the second derivative of $T$ required evaluated at $(u, v)=(0,0)$ is $4\left(a_{0}^{3}-c_{0}\right)\left(a_{0}-a_{2}\right)-b_{1}^{2}=0$, where $C=\sum_{i=0}^{3} b_{i} u^{3-i} v^{i}+\sum_{i=0}^{4} c_{i} u^{4-i} v^{i}$ (note that $b_{0}=0$ from above). On the other hand in the 4-jet of the distance-squared function from $\left(0,0,1 / 2 a_{0}\right)$ we know that the $u^{3}$ term is zero, and the only terms required to determine if we have an $A_{\geq 4}$ are those of $v^{2}, u^{2} v$ and $u^{4}$. These are easily found to be $\left(1-a_{2} / a_{0}\right) v^{2}$, $\left(-b_{1} / a_{0}\right) u^{2} v,\left(a_{0}^{2}-c_{0} / a_{0}\right)$ and we have an $A_{\geqq 4}$ if and only if their sum is a perfect square. This yields the same condition as above.

Now for the proof of (ii). Here the tangency condition is equivalent to the second derivative $T_{u v}$ vanishing. Using the equation $P(u, v, T(u, v))=0$ it is not difficult to see that this second order derivative vanishes if and only if $P_{t} P_{u v}-P_{v} P_{u t}=0$. Setting $t=2 a_{2}$ we obtain $8\left(a_{2}-a_{0}\right)\left\{3\left(a_{0}-a_{2}\right) c_{3}-b_{1} b_{2}+3 b_{2} b_{3}\right\}$. In this case we have (from the results in the first part of the paper) $b_{2}=0$, and the condition comes down to $c_{3}=0$. If we now consider the 4 -jet of the folding map we obtain

$$
\left(u^{2}, v, a_{0} u^{2}+a_{2} v^{2}+b_{0} u^{3}+b_{1} u^{2} v+b_{3} v^{3}+c_{0} u^{4}+\cdots+c_{4} v^{4}\right)
$$

An obvious change of co-ordinates in the target reduces this to

$$
\left(u^{2}, v, b_{0} u^{3}+c_{1} u^{3} v+c_{3} u v^{3}\right)
$$

which is of type $S_{\geqq 3}$ if and only if $c_{3}=0$.

\section{REFERENCES}

1. J. W. Bruce, Generic reflections and projections, Math. Scand. 54 (1984), 262-278.

2. J. W. BruCE and T. C. WILkinson, Folding maps and focal sets, in Proceedings of Warwick Symposium on Singularities (Springer Lecture Notes in Math., 1462 Springer-Verlag, Berlin and New York, 1991), 63-72.

3. A. P. Gourdon, Face recognition from depth maps and surface curvature, in Proceedings of SPIE Conference on Geometric Methods in Computer Vision (San Diego, CA, July 1991).

4. A. Guéżec, Large deformable splines, crest lines and matching, INRIA, November 1992, preprint.

5. M. M. LIPSChUTZ, Differential Geometry (Schaum's Outline Guide Series, McGraw-Hill, New York, 1969). 
6. D. M. Q. Mond, On the classification of germs of maps from $\mathbf{R}^{2}$ to $\mathbf{R}^{3}$, Proc. London Math. Soc. 50 (1985), 333-369.

7. R. J. Morris, Symmetry of curves and the geometry of surfaces: two explorations with the aid of computer graphics (Thesis, University of Liverpool, 1991).

8. I. R. Porteous, The normal singularities of a submanifold, J. Differential Geom. 5 (1971), 543-564.

9. I. R. Porteous, The normal singularities of surfaces in $\mathbf{R}^{3}$, in Proceedings of Symposia in Pure Mathematics (Volume 40, Part 2, American Mathematical Society, 1983, Providence), 379-394.

10. 1. R. Porteous, Geometric Differentiation (Cambridge University Press, Cambridge, 1994).

11. J. -P. Thirion and A. Gourdon, The 3D marching lines algorithm, INRIA, preprint.

12. J. -P. ThIRION and A. Gourdon, The marching lines algorithm: new results and proofs (INRIA research report No. 1881, March 1993).

13. C. T. C. WALL, Geometric properties of generic differentiable manifolds, in Geometry and Topology, Rio de Janeiro 1976 (Lecture Notes in Mathematics 597, 707-774, Springer-Verlag, New York), 707-775.

14. T. C. Wilkinson, The geometry of folding maps (Thesis, University of Newcastle-upon-Tyne, 1991).

Department of Pure Mathematics

ThE UnIVERSITY

P.O. Box 147

LIVERPOOL L69 3BX

U.K. 